\title{
REMOÇÃO DE COR E MATÉRIA ORGÂNICA DE LIXIVIADO DE ATERRO SANITÁRIO POR MEIO DE TRATAMENTO COM O $\mathrm{O}_{3}, \mathrm{O}_{3} / \mathrm{TiO}_{2}$ e $\mathrm{O}_{3} / \mathrm{ZnO}$
}

\author{
B. L. CORSO ${ }^{1}$, A. P. J. SCANDELAI ${ }^{1}$, C. R. G. TAVARES ${ }^{1}$ \\ ${ }^{1}$ Universidade Estadual de Maringá, Departamento de Engenharia Química \\ E-mail para contato: beatrizlcorso@gmail.com
}

\begin{abstract}
RESUMO - O lixiviado, gerado pela degradação dos resíduos sólidos, apresenta alta concentração de matéria orgânica de difícil remoção por processos convencionais e biológicos. O presente trabalho objetivou avaliar a eficiência da ozonização e ozonização catalítica $\left(\mathrm{O}_{3} / \mathrm{TiO}_{2}, \mathrm{O}_{3} / \mathrm{ZnO}\right)$ na remoção de cor e matéria orgânica presentes no lixiviado gerado no aterro sanitário de Maringá-PR. A eficiência dos tratamentos foi avaliada por meio da remoção de cor aparente e matéria orgânica [DQO (demanda química de oxigênio), DBO (demanda bioquímica de oxigênio) e COD (carbono orgânico dissolvido)]. Os tratamentos apresentaram altas remoções de cor $(>93 \%)$, porém baixas remoções de matéria orgânica, indicadas pela remoção de DQO, DBO e COD.
\end{abstract}

\section{INTRODUÇÃO}

O lixiviado, líquido gerado no processo de degradação da matéria orgânica sólida em aterros sanitários (Coelho et al., 2012), possui coloração escura, odor desagradável, é constituído de substâncias orgânicas e inorgânicas e sua composição é variável, dependendo das características dos resíduos depositados e das condições ambientais (Morais, 2005; Kjeldsen et al., 2002). Em aterros jovens ( $<5$ anos), como o aterro em estudo, em geral, o lixiviado apresenta alta concentração de matéria orgânica refratária e elevada cor (Bidone, 2007).

Por conter compostos de difícil degradação, os processos convencionais não tem se mostrado satisfatórios para degradação desse efluente (Pedroso, 2012). Sendo assim, os Processos Oxidativos Avançados (POA) têm sido utilizados no tratamento de efluentes como o lixiviado, por serem bastante eficientes na destruição de substâncias orgânicas de difícil degradação (Coelho et al., 2012). Nestes processos, os elétrons são removidos de uma substância aumentando seu estado de oxidação e podem ser considerados como tecnologias limpas, pois não há formação de subprodutos sólidos (lodo), podem gerar compostos mais facilmente degradáveis e os produtos finais da reação são $\mathrm{CO}_{2}$ e $\mathrm{H}_{2} \mathrm{O}$ (Ferreira et al., 2001). Segundo EPA (1998) a formação do radical hidroxil $(\bullet \mathrm{OH})$ desses processos pode ser intensificada com a adição de catalisadores, que removem compostos refratários à ozonização convencional. Dentre os óxidos metálicos utilizados na ozonização catalítica heterogênea, o dióxido de titânio $\left(\mathrm{TiO}_{2}\right)$ e o óxido de zinco $(\mathrm{ZnO})$ tem se destacado por apresentarem alta atividade catalítica, não serem tóxicos e apresentarem custo relativamente baixo (Assalin e Durán, 2006; Naik e Fernandes, 1999). 
Neste contexto, o objetivo desse trabalho foi avaliar a eficiência dos POA ozonização $\left(\mathrm{O}_{3}\right)$ e ozonização catalítica $\left(\mathrm{O}_{3} / \mathrm{TiO}_{2}, \mathrm{O}_{3} / \mathrm{ZnO}\right)$ na remoção de cor e matéria orgânica contidas no lixiviado gerado no aterro sanitário de Maringá-PR.

\section{MATERIAIS E MÉTODOS}

\subsection{Sistema de Ozonização e Tratamentos}

Os experimentos foram realizados em um sistema de ozonização constituído por um reator em coluna, um concentrador de oxigênio $\left(\mathrm{O}_{2}\right)$ e um gerador de ozônio $\left(\mathrm{O}_{3}\right)$. O reator foi operado descontinuamente (batelada) e a distribuição de $\mathrm{O}_{3}$ se deu por placas porosas fixadas na base da coluna. $\mathrm{O}$ sistema de fornecimento do $\mathrm{O}_{3}$ consistia em um concentrador de $\mathrm{O}_{2}$ (responsável por produzir oxigênio concentrado com alta pureza, a partir do ar ambiente) acoplado ao gerador de $\mathrm{O}_{3}$ (responsável pela transformação do $\mathrm{O}_{2}$ em $\mathrm{O}_{3}$, por meio de descargas elétricas). $\mathrm{O}$ concentrador de $\mathrm{O}_{2}$ foi operado com vazão de $4 \mathrm{~L} \mathrm{O}_{2}$ minn $^{-1}$, gerando 3,98 $\mathrm{g} \mathrm{O}_{3} \mathrm{~h}^{-1}$. Para cada ensaio foi utilizado um volume fixo de 5 litros de lixiviado e tempo de reação de 90 minutos. Os tratamentos utilizados foram: $\mathrm{O}_{3}, \mathrm{O}_{3}+0,5 \mathrm{~g} \mathrm{TiO}_{2} \mathrm{~L}^{-1}$ e $_{3}+0,5 \mathrm{~g}$ $\mathrm{ZnO} \mathrm{L}{ }^{-1}$, todos com o $\mathrm{pH}$ original do efluente, próximo do neutro.

\subsection{Coleta, Preservação e Caracterização do Lixiviado}

As amostras de lixiviado foram coletadas no tanque de armazenamento de lixiviado, instalado no aterro sanitário de Maringá-PR e conduzidas ao Laboratório de Gestão, Controle e Preservação Ambiental (LGCPA) da Universidade Estadual de Maringá (UEM), no qual os ensaios foram realizados. Preliminarmente, o lixiviado foi caracterizado por meio da determinação dos seguintes parâmetros: cor aparente, Demanda Química de Oxigênio (DQO), Demanda Bioquímica de Oxigênio $\left(\mathrm{DBO}_{5}{ }^{20}\right)$ e Carbono Orgânico Dissolvido (COD).

\subsection{Controles Analíticos}

A cor aparente foi determinada em espectrofotômetro $\mathrm{HACH}$, modelo $\mathrm{DR} / 2010$, pelo método platina-cobalto (HACH, 1996 - Método 8025). A determinação da DQO foi realizada pelo método colorimétrico, de acordo com a metodologia descrita no Standard Methods for the Examination of Water and Wastewater (APHA, 1998 - Método 5220D) e a amostra foi previamente filtrada em membrana de poro $0,45 \mu \mathrm{m}$ para reduzir a interferência dos catalisadores utilizados nos tratamentos. A $\mathrm{DBO}_{5}{ }^{20}$ foi determinada pelo método respirométrico (APHA, 1998 - Método 5210 D), por meio da incubação da amostra por 5 dias a $20{ }^{\circ} \mathrm{C}$. O COD foi determinado em um analisador espectrofotométrico Shimadzu, modelo TOC-L CPH, pelo método de combustão infravermelha (APHA, 1998 - Método 5310 B). Estes procedimentos foram utilizados tanto para caracterização das amostras de lixiviado bruto como para os controles dos tratamentos de ozonização e ozonização catalítica. 


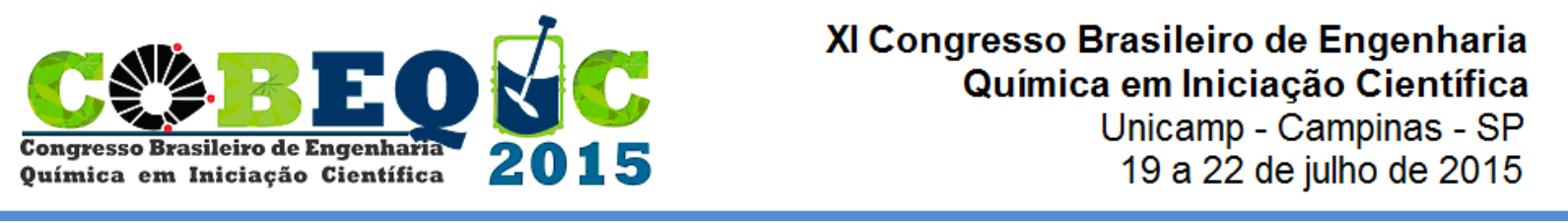

\section{RESULTADOS E DISCUSSÃO}

\subsection{Cor Aparente}

Os resultados obtidos e apresentados na Tabela 1 mostraram uma elevada remoção percentual de cor aparente, indicando a eficiência dos três tratamentos para a descoloração. $\mathrm{O}$ tratamento utilizando apenas $\mathrm{O}_{3}$ se destacou dos demais apresentando uma remoção percentual de $97,4 \%$, devido ao ataque direto do ozônio aos compostos aromáticos ou com ligações duplas que são responsáveis pela sua coloração.

Segundo Von Sperling (2014) a coloração escura do lixiviado pode advir de metais como ferro ou manganês, ou ainda devida à existência de matérias em suspensão. Por meio da Figura 1 é possível observar a mudança de coloração do lixiviado bruto (amostra 1) após os tratamentos utilizando apenas ozonização (amostra 2), ozonização catalítica com $\mathrm{TiO}_{2}$ (amostra 3) e ozonização catalítica com ZnO (amostra 4).

Tabela 1 - Valores de cor aparente no lixiviado bruto e após os tratamentos

\begin{tabular}{ccc}
\hline Tratamento & Cor Aparente $\left(\mathrm{mg} \mathrm{PtCo} \mathrm{L}^{-1}\right)$ & $\%$ de remoção \\
\hline Lixiviado Bruto & 5540 & - \\
$\mathrm{O}_{3}$ & 145 & 97,4 \\
$\mathrm{O}_{3}+0,5 \mathrm{~g} / \mathrm{L} \mathrm{TiO}_{2}$ & 180 & 96,8 \\
$\mathrm{O}_{3}+0,5 \mathrm{~g} / \mathrm{L} \mathrm{ZnO}$ & 350 & 93,7 \\
\hline
\end{tabular}

Figura 1 - Remoção de cor aparente após os tratamentos

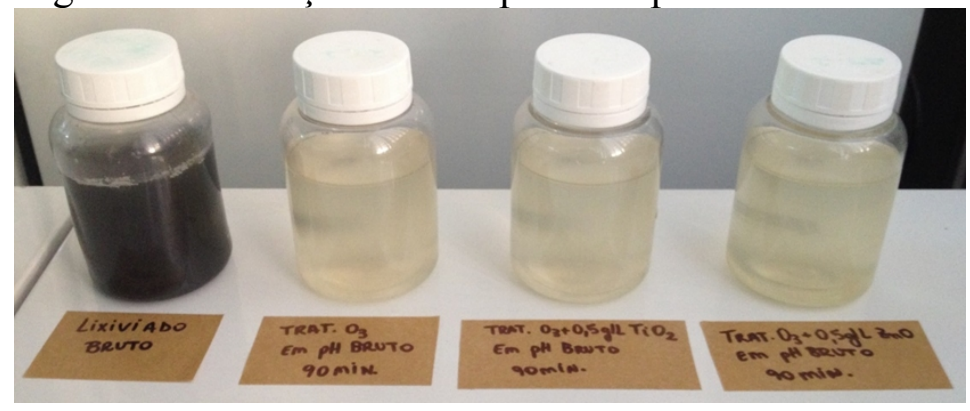

\subsection{Demanda Química de Oxigênio (DQO)}

Os tratamentos utilizados apresentaram baixas remoções percentuais de matéria orgânica, indicada pela redução da DQO (Tabela 2). Contudo, o tratamento utilizando ozonização catalítica com $\mathrm{TiO}_{2}$, foi o que apresentou melhor resultado, apresentando uma remoção percentual de 32,7\%. Telles (2010) também obteve uma baixa eficiência na remoção da DQO (13\%) para o lixiviado de aterro sanitário tratado apenas com ozonização.

Uma hipótese para esses resultados é a formação de compostos secundários durante o processo de ozonização, constituintes inorgânicos como ferro II $\left(\mathrm{Fe}^{2+}\right)$, manganês II $\left(\mathrm{Mn}^{2+}\right)$, 


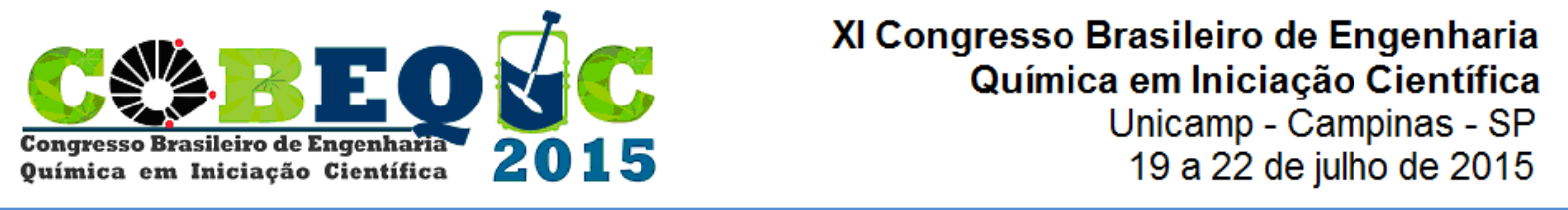

sulfetos $\left(\mathrm{S}^{2+}\right)$ e cloretos $\left(\mathrm{Cl}^{-}\right)$(Morais, 2005; Kjeldsen et al., 2002) que estariam interferindo na faixa de leitura.

Tabela 2 - Valores de DQO no lixiviado bruto e após os tratamentos

\begin{tabular}{ccc}
\hline Tratamento & DQO $\left(\mathrm{mg} \mathrm{O}_{2} \mathrm{~L}^{-1}\right)$ & \% de remoção \\
\hline Lixiviado Bruto & 4263,0 & - \\
$\mathrm{O}_{3}$ & 3628,5 & 14,9 \\
$\mathrm{O}_{3}+0,5 \mathrm{~g} / \mathrm{L} \mathrm{TiO}_{2}$ & 2868,0 & 32,7 \\
$\mathrm{O}_{3}+0,5 \mathrm{~g} / \mathrm{L} \mathrm{ZnO}$ & 3661,8 & 14,1 \\
\hline
\end{tabular}

\subsection{Demanda Bioquímica de Oxigênio $\left(\mathrm{DBO}_{5}{ }^{20}\right)$}

Como apresentado na Tabela 3, os tratamentos propostos apresentaram baixa remoção percentual de matéria orgânica, indicada pela redução da $\mathrm{DBO}_{5}{ }^{20}$. O tratamento utilizando apenas ozonização apresentou a maior remoção percentual, $38,7 \%$, e o tratamento com $\mathrm{ZnO}$ apresentou a menor eficiência de remoção, o que indica que sua utilização nas condições estabelecidas não favoreceu à remoção de matéria orgânica.

As baixas remoções de $\mathrm{DBO}_{5}{ }^{20}$ podem ser atribuídas, segundo Telles (2010), pela não oxidação de compostos biodegradáveis pelo ozônio ou ainda pela formação de substâncias com alguma toxicidade para os microrganismos presentes no ensaio de $\mathrm{DBO}_{5}{ }^{20}$.

Tabela 3 - Valores para a DQO no lixiviado bruto e após os tratamentos

\begin{tabular}{ccc}
\hline Tratamento & $\mathrm{DBO}_{5}{ }^{20}\left(\mathrm{mg} \mathrm{O}_{2} \mathrm{~L}^{-1}\right)$ & \% de remoção \\
\hline Lixiviado Bruto & 395 & - \\
$\mathrm{O}_{3}$ & 242 & 38,7 \\
$\mathrm{O}_{3}+0,5 \mathrm{~g} / \mathrm{L} \mathrm{TiO}_{2}$ & 262 & 33,7 \\
$\mathrm{O}_{3}+0,5 \mathrm{~g} / \mathrm{L} \mathrm{ZnO}$ & 379 & 4,1 \\
\hline
\end{tabular}

\subsection{Carbono Orgânico Dissolvido (COD)}

A remoção percentual da matéria orgânica, indicada pela redução do COD, foi baixa nas condições utilizadas, como pode ser visto na Tabela 4. O tratamento que apresentou melhor remoção foi aquele que utilizou apenas ozonio, 38,7\%. Esses valores indicam que os tratamentos utilizados não levaram à uma mineralização significativa dos compostos orgânicos presentes no lixiviado, devendo ser avaliadas novas condições experimentais.

Segundo Bila et al., (2005), no caso de efluentes de elevada recalcitrância como o lixiviado, a ozonização pode ser utilizada em conjunto com um processo biológico, tornando o processo de assimilação da matéria orgânica mais fácil e com a vantagem de não promover a geração de lodo. 
Tabela 4 - Valores de COD no lixiviado bruto e após os tratamentos

\begin{tabular}{ccc}
\hline Tratamento & $\mathrm{COD}\left(\mathrm{mg} \mathrm{L}^{-1}\right)$ & \% de remoção \\
\hline Lixiviado Bruto & 11360 & - \\
$\mathrm{O}_{3}$ & 6966 & 38,7 \\
$\mathrm{O}_{3}+0,5 \mathrm{~g} / \mathrm{L} \mathrm{TiO}_{2}$ & 7084 & 37,6 \\
$\mathrm{O}_{3}+0,5 \mathrm{~g} / \mathrm{L} \mathrm{ZnO}$ & 7398 & 34,9 \\
\hline
\end{tabular}

\section{CONCLUSÃO}

Como os resultados obtidos permitiram observar, os tratamentos por ozonização e ozonização catalítica com $\mathrm{TiO}_{2}$ e $\mathrm{ZnO}$ foram muito eficientes na descoloração do lixiviado, porém menos eficientes para a remoção da matéria orgânica nas condições utilizadas. Este pode ser um indício de que os tratamentos estudados seriam mais eficazes se combinados a outros processos de degradação, utilizando-os como pré ou pós-tratamento, sendo uma alternativa aos processos convencionais de tratamento do lixiviado.

\section{REFERÊNCIAS}

APHA - AMERICAN PUBLIC HEALTH ASSOCIATION. Standard methods for the examination of water and wastewater. 20 ed. APHA: USA, 1998.

ASSALIN, M. R.; DURÁN, N. Novas tendências para aplicação de ozônio no tratamento de resíduos: ozonização catalítica. Revista Analytica, n. 26, p. 76-86, 2006.

BIDONE, R. F. Tratamento de Lixiviado de Aterro Sanitário por um Sistema Composto por Filtros Anaeróbios seguidos de Banhados Construídos: Estudo de caso - Central de Resíduos de Recreio em Minas do Leão/RS. Dissertação (Mestrado em Hidráulica e Saneamento), EESC/USP, São Paulo, 2007.

BILA, D. M.; MONTALVÃO, A. F.; SILVA A. C.; DEZOTTI, M. Ozonation of a landfill leachate: evaluation of toxicity removal and biodegradability improvement. J. Hazard. Mater., v. B117, p. 235-242, 2005.

COElHO, M. G.; SANTOS, L. P.; SANTOS, C. L.; ANDRADE, S. M. A. Utilização de processos oxidativos avançados (POAs) no tratamento de líquidos percolados provenientes do aterro sanitário de Uberlândia-MG/Brasil. In: Anais do XXVIII Congresso Interamericano de Engenharia Sanitária e Ambiental, Cancún, 2002.

EPA - ENVIRONMENTAL PROTECTION AGENCY. Advanced Photochemical Oxidation Processes, Handbook, Washington, U.S., 1998.

FERREIRA, J. A.; GIORDANO, G.; RITTER, E.; ROSSO, T. C. A.; CAMPOS, J. C.; LIMA, P. Z. M. Uma revisão das técnicas de tratamento de chorume e a realidade do Estado do Rio de Janeiro. In: $21^{\circ}$ Congresso Brasileiro de Engenharia Sanitária e Ambiental, João Pessoa, PB, 2001.

HACH Company. Procedures Manual of Spectrophotometer DR/2010. USA, 1996. 
KJELDSEN, P.; BARLAZ, M. A.; ROOKER, A. P.; BAUN, A.; LEDIN, A.; CHRISTENSEN, T. Present and long-term composition of MSW landfill leachate: a review. Environ. Sci. Technol., v. 32, n. 4, p. 297-336, 2002.

MORAIS, J. L. Estudo da potencialidade de processos oxidativos avançados, isolados e integrados com processos biológicos tradicionais, para tratamento de chorume de aterro sanitário. Tese (Doutorado em Química), UFPR, Curitiba, PR, 2005.

NAIK, S. P.; FERNANDES, J. B. Temperature programmed desorption studies on a new active zinc oxide catalyst. Thermochim. Acta, v. 332, p. 21-25, 1999.

PEDROSO, K. Avaliação do tratamento do lixiviado do aterro sanitário de Maringá, Paraná, por processo de coagulação/floculação e ozonização. Dissertação (Mestrado em Engenharia Urbana), UEM, Maringá, PR, 2012.

TELLES, C. A. S. Processos combinados para o tratamento de lixiviado de aterro sanitário. Dissertação (Mestrado em Engenharia Química), UFRJ, Rio de Janeiro, RJ, 2010.

VON SPERLING, M. Introdução à qualidade das águas e ao tratamento de esgotos. 4 ed. Belo Horizonte: Editora UFMG, 2014. 